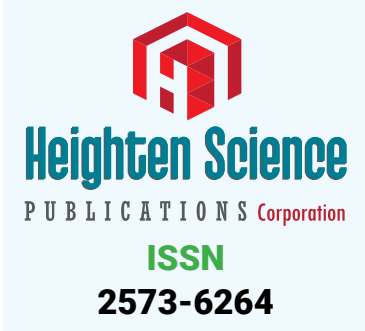

*Address for Correspondence: Azzam Alarab, Department of Physiotherapy, Faculty of Applied Medical Health, Palestine Ahlyia University, Dheisha, Bethlehem, Palestine, Tel: +009702751566; Ext: 213;

Email: azzam@paluniv.edu.ps

Submitted: 01 April 2019

Approved: 22 April 2019

Published: 23 April 2019

Copyright: (c) 2019 Falso M, et al. This is an open access article distributed under the Creative Commons Attribution License, which permits unrestricted use, distribution, and reproduction in any medium, provided the original work is properly cited

Keywords: Lteral epicondalyitis; Ultrasound; Kinesio tape; Pain intensity; Hand grip strength
Research Article

\section{Effectiveness of therapeutic ultrasound and kinesio tape in treatment of tennis elbow}

\author{
Hamza Shaheen ${ }^{1}$, Azzam Alarab ${ }^{1 *}$ and Muntaser S Ahmad ${ }^{1,2}$ \\ 'Department of Physiotherapy, Faculty of Applied Medical Health, Palestine Ahlyia University, \\ Dheisha, Bethlehem, Palestine \\ 2Department of Medical Physics and Radiation Science, School of Physics, Univirsti Sains \\ Malaysia, 11800 Penang, Malaysia
}

\section{Abstract}

Objective: To investigate the effect of kinesio tape and therapeutic ultrasound on pain and hand grip strength associated with tennis elbow.

Material and Methods: Twenty male and female patients with age from 20-50 years suffering from lateral epicondylitis were participated in this study. All patients were randomly selected from orthopaedic surgeon. They were dividing into two groups. Group (A) (ultrasound and exercises group). Group (B) (kinesio tape and exercises group). The hand-held dynamometer was used to provide a detailed and objective measurement of wrist joint strength throughout its range of motion, the pressure being registered in kilogram (Kg) and Visual Analogue Scale (VAS) was used to record the degree of pain intensity.

Result: The result shows no significant difference between group $A$ and $B$ in their ages, weights, heights, and $B M I$ where their $t$ and $P$-values were $(0.8,0.43),(0.03,0.97),(0.98,0.33)$, and $(0.9,0.37)$ respectively. In Group $A$ the significant differences in hand grip strength between pre and post-test which shows 31.59), compared to the pre-test (27.35), while in group B (33.17) compared to the pre-test (23.88). According the pain scale for group A and group B there are significant differences where the results were (2.50), compared to the pre-test (5.90) and (2.00), compared to the pre-test (7.30), retrospectively.

Conclusion: The treatment was improvement between both groups. However, the kinesio tape is better than therapeutic ultrasound.

\section{Introduction}

Lateral epicondylitis is considered one of the painful condition that effects on the tendons tissue at the wrist extensor muscles origin, which leads to function losing of the affected limb [1]. Tennis elbow starts as a micro-tear of the wrist extensor muscles origin which affected on the granulation tissue formation. The affected tendons would not be able to do the activity as well functions which involve wrist extension, repeated gripping, forearm pronation and supination. The resulting of this mechanical failure are consist of tendinitis and tennis elbow symptoms such as lateral epicondyle pain of humorous [2]. The complication of the tennis elbow starts after 24-72 hours which occurs after unaccustomed activity such as repeating wrist extension.

There are different theoretical mechanisms to treat the tennis elbow and these mechanisms have the same goal representing on pain reducing and functional improvement. The ultrasound, friction massage, strengthening exercise, stretching, and electrical stimulation are the most common modalities which used to rehabilitee the tennis elbow $[3,4]$. The strengthening and stretching programs are the most 
common therapy program used, and the physiotherapists used different modalities in this program such as hyperthermia, iontophoresis, splinting, low-level laser therapy, and kinesio taping $[5,6]$.

Therapeutic ultrasound is widely used practice in physiotherapy and sport medicine to treat different injures, and this method is focused on the changing the extensibility of the collagenous tissues to improve range of motion [7]. The kinesio taping is considered as one of the stretching exercises and it used to provide supporting and protection for the joint with permitting the optimal movement for it, thus the stability of joint would be increased. However, the taping may cause some proprioceptive influences including cutaneous stimulation and enhanced awareness of shoulder girdle and upper trunk position [8,9]. In the present work, the therapeutic effectiveness of ultrasound and kinesio tape in minimizing pain intensity and improving hand griping strength in tennis elbow cases were investigated.

\section{Materials and Methods}

\section{Subjects}

Totally 20 patients (male: 10 , female: 10 ) with age between $20-50$ years (mean age 25.35 \pm 3.25 years) were diagnosed with lateral epicondylitis based on confirmed inclusion and exclusion criteria that assigned into two groups, all patients were randomly selected from orthopedic surgeon, and they presenting to the Palestine Ahliya University, Physical Therapy Clinic at the between Dec, 2018 and Jan, 2019 were considered. Group-A (ultrasound and exercises group) and Group-B (kinesio tape and exercises group) provided with 10 patients received ultrasound combined with exercises for 12 sessions over a four weeks period, three sessions each week and 10 patients received kinesio tape combined with exercises for 12 sessions over a four weeks period, three sessions each week, respectively.

Screening was performed by 2 physical therapists, both of them with minimum 4 years of clinical experience in outpatient orthopaedic settings. Upon the exam, the inclusion criteria included: (a) age ranged from 20 to 50 years; (b) chronic pain over lateral epicondyle; and (c) dominant hand affected. The exclusion criteria were (a) patients with polyarthritis, carpal tunnel syndrome, rotator cuff tendonitis, cervical spondylosis and neurological abnormalities in affected area; (b) osteoporotic and rheumatoid arthritis patients; (c) Old fracture at the affected arm; and (d) serious elbow injury at the time of treatment. The exclusion criteria were picked to increase the selected subject's homogeneity, and to preclude the pathology that are likely to be less responsive to the selected taping intervention. The patients (subjects) were given a verbal description and informed written consent before the participation.

\section{Instruments}

\section{Instruments for assessment}

The study was used two devices to assess the treatment effectiveness in both groups.

Hand held dynamometer: The hand-held dynamometer was utilized to provide a wrist extension strength during the wrist motion, and the pressure being registered in kilogram (Kg).

Visual Analogue Scale (VAS): Pain was measured on a visual analogue scale (VAS), where $0(\mathrm{~cm})$ was least pain imaginable and $10(\mathrm{~cm})$ was worst pain imaginable, each subject was asked to mark and score on the line at the point that representing her intensity of pain [10].

\section{Instruments for treatment}

Ultrasound: Ultrasound waves are generated by a piezoelectric effect caused by 
vibration of crystals within the head of the wand/probe. The sound waves that pass through the skin cause vibration of the local tissue. This vibration or cavitations can cause a deep heating locally though usually no sensation of heat is felt by the patient, frequency of therapeutic ultrasound ranges from 0.5-3.0 MHZ.

Kinesio tape: Group-B (Elbow Taping): this group were received a standardized therapeutic Kinesio Tape (KT) application (Figure 1). The taping technique consisted of $10-15 \mathrm{~cm}$ long, $5 \mathrm{~cm}$ in width with strong adhesive mass, and $5 \mathrm{~cm}$ wide cohesive bandage of Johnsonplast ${ }^{\mathrm{TM}}$. Elbow taping was fixed in the fit place in all treatment session, expect if there was an adverse skin reaction.

\section{Procedures}

Assessment procedures: Each subject had VAS and hand grip measures completed before and after the initial tape application, and all of patients were informed about the study steps. The pain intensity assessment was performed by Thomsen test by active dorsiflexion of the wrist of the affected arm against resistance with the patient comfortably sitting, elbow extended and forearm pronated. while the hand grip was performed by the hand-held dynamometer, the subject was sit with their shoulder adducted and neutrally rotated, elbow flexed at $90^{\circ}$, forearm in neutral position, and wrist between $0^{\circ}$ and $30^{\circ}$ dorsiflexion and between $0^{\circ}$ and $15^{\circ}$ ulnar deviation. After the individual is positioned properly, say, "Squeeze as hard as you can ...harder! harder! relax." Record the scores of three successive trials for hand tested, the average of the pressure being registered in $\mathrm{Kg}$.

Treatment procedures: Each group were given different procedures. For Group (A) the patient was sit with the elbow flexed; forearm pronated and supported. $1 \mathrm{Mz}$, continuous mode of application, intensity level $1.5 \mathrm{w} / \mathrm{cm}, 5$ minutes/session, 12 sessions for four weeks, 3 sessions /week treatment diameters, while Group B the tape was applied with the affected muscle in a stretched position, taping from the origin of the muscle to the insertion point. However, both subjects were received progressive strengthening and stretching exercises programme based on the work of previous study [11]. Exercises performed for both groups included: Four-step exercises programme. Each step will be performed in one week for four weeks:-

Step 1: slow fist clenching; The patient was asked to clench his hand eight counts (times) after sitting position, elbow extended, forearm rested on the treatment table and comfortably pronated, and then the patient was asked to extend the wrist counting to eight after the therapists applied the resistance force for the patient hand including dorsal surface and palmar surface.

Step 2: exercises against an elastic band for wrist flexion; The patient was instructed to flex his wrist and make the ulnar and radial deviate during the holding the other end of the band and that happened after patients sitting position, elbow semi flexed, forearm supinated and rested on the treatment table.

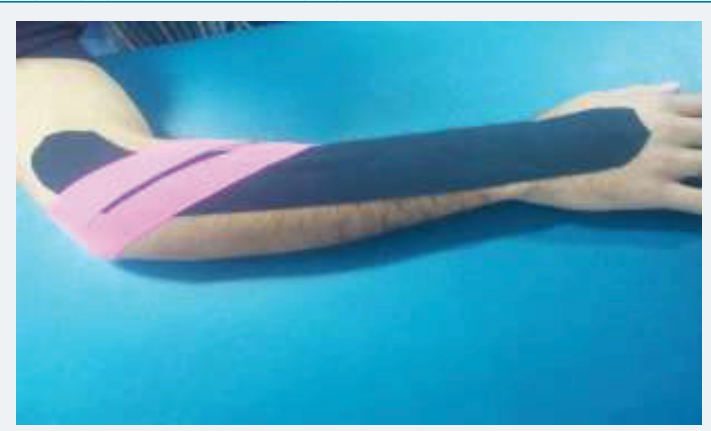

Figure 1: Elbow kinesio taping 
Step 3: Wrist extension/ flexion with ulnar and radial deviation against resistance; The patient was instructed to extend his wrist with ulnar and radial deviation toward little finger, and that happened after the Patient in sitting position, elbow semi flexed, forearm supinated and rested on the treatment table, and the investigator's one hand supports the wrist and the other hand is placed on the palmer surface at side of the thumb finger and pushes the hand upward toward the little finger.

Step 4: soft ball compressing exercises and twisting a towel into a roll; the patient was instructed to compress the soft ball and twist a towel into a roll with both hands.

\section{Data analysis}

For assessment of effect of counterforce forearm brace in pre and post intervention mean scores were analysed using a paired-sample t-test to determine whether there were any significant differences. Statistical analysis was performed with SPSS (version 15.0), with P-values less than 0.05 considered statistically significant.

\section{Results}

A total of 20 patients with tennis elbow based on the inclusion criteria (10 men and 10 women with mean ages $25.35 \pm 3.25$ years) were involved in the study. 17 individuals were right dominant hand and 3 individuals were left-handed. Mean $( \pm S D$ ) duration of their tennis elbow condition was $5.1 \pm 1.1$ weeks. Randomly chosen for patients and classified into two groups.

\section{Group (A)}

Ten patients were included in this group. The data in figure 2 represented their mean age $(26.90 \pm 8.157)$ years, mean weight $(68.50 \pm 11.167)$ kilograms $(\mathrm{Kg})$, mean height $(1.709 \pm 0.092)$ centimetres $(\mathrm{cm})$, and mean BMI $(0.043 \pm 0.007) \mathrm{Kg} / \mathrm{m}^{2}$.

Table 1 and Figure 3 shows the results of the hand grip strength for group A and it indicated that there are significant differences between the pre and posttest, and the differences were for the post test, which shows more grip strength by mean equal to (31.59), compared to the pre-test (27.35).

Table 2 and Figure 4 shows the results of the Pain strength for group A and it the results indicated that there are significant differences between the pre and post-test, and the differences were for the post test, which shows less pine by mean equal to (2.50), compared to the pre-test (5.90).


Figure 2: The sample in group A; (a) the main age; (b) main weight; (c) main height; and (d) the main BMI of the group. 
Table 1: Paired sample t-test for the comparisons in Hand grip strength for group A.

\begin{tabular}{|c|c|c|c|c|c|c|}
\hline Test & Number & Mean & Std. Deviation & Df & t-value & p-value \\
\hline Pre & 10 & 27.35 & 14.11 & \multirow{2}{*}{9} & -5.382 & 0.000 \\
\hline Post & 10 & 31.59 & 14.47 & & \\
\hline
\end{tabular}

Table 2: Paired sample t-test for the comparisons in the Pain for group A.

\begin{tabular}{|c|c|c|c|c|c|c|}
\hline Test & Number & Mean & Std. Deviation & Df & t-value & p-value \\
\hline Pre & 10 & 5.90 & 1.96 & 9 & 10.002 & 0.000 \\
\hline Post & 10 & 2.50 & 1.84 & & & 0.000 \\
\hline
\end{tabular}
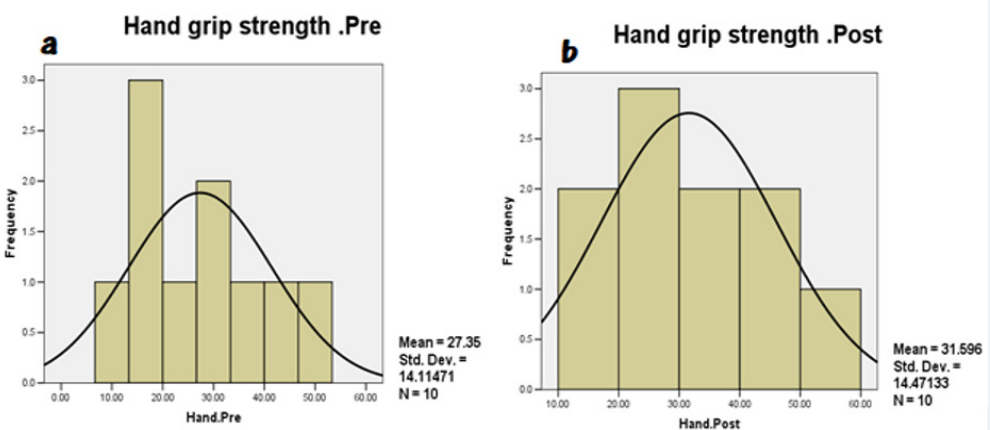

Figure 3: The effectiveness of the treatment for the hand grip in group A; (a) pre- treatment; and (b) post treatment
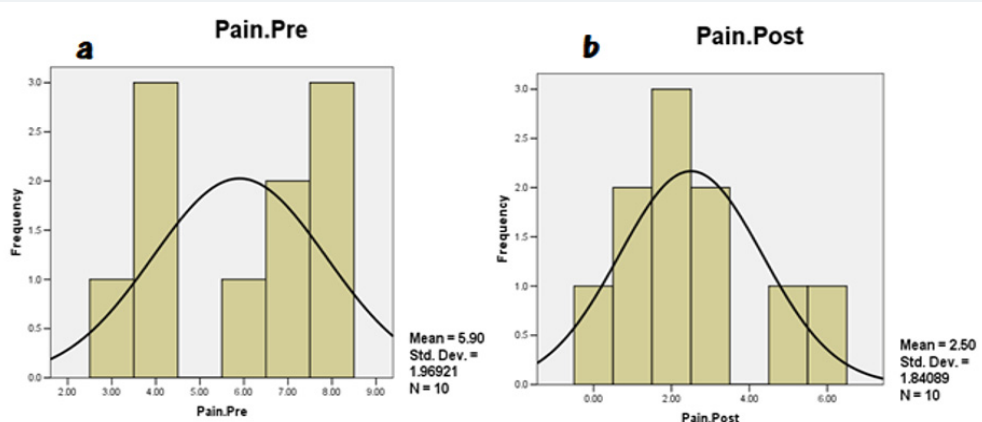

Figure 4: The defferences of the pain for the group A between; (a) pre-treatment; and (b) post treatment.

\section{Group (B)}

Ten patients were included in this group. The data Figure 5 represented their mean age (38.60. \pm 8.934$)$ years, mean weight $(87.90 \pm 18.18)$ kilograms $(\mathrm{Kg})$, mean height $(1.725 \pm 0.072)$ centimetres $(\mathrm{cm})$, and mean BMI $(0.034 \pm 0.006) \mathrm{Kg} / \mathrm{m}^{2}$.

Figure 6 shows the results of the hand grip strength for group B and it indicated that there are significant differences between the pre and post-test, and the differences were for the post test, which shows more grip strength by mean equal to (33.17), compared to the pre-test (23.98).

Figure 7 shows the results of the Pain strength for group B and it indicated that there are significant differences between the pre and post-test, and the differences were for the post test, which shows less pine by mean equal to 2 , compared to the pretest (7.3).

\section{Summary}

There was no significant difference between both groups in their ages, weights, heights, and BMI where their $t$ and P-values were $(0.8,0.43),(0.03,0.97),(0.98,0.33)$, and $(0.9,0.37)$ respectively. There was significant difference between group $(A)$ and group (B) as regard to pain reduction. Also, there was significant difference between 
(A) and (B) as regard to grip strength improvement in both groups, which (B) allows a greater degree of pain relief and allows a greater improvement in grip strength than (A).

The following figure 8 shows the summary of the result between both groups.

\section{Discussion}

The purpose of this study was comparison between the effects of therapeutic ultrasound and kinesio taping on improvement of pain intensity and hand grip strength

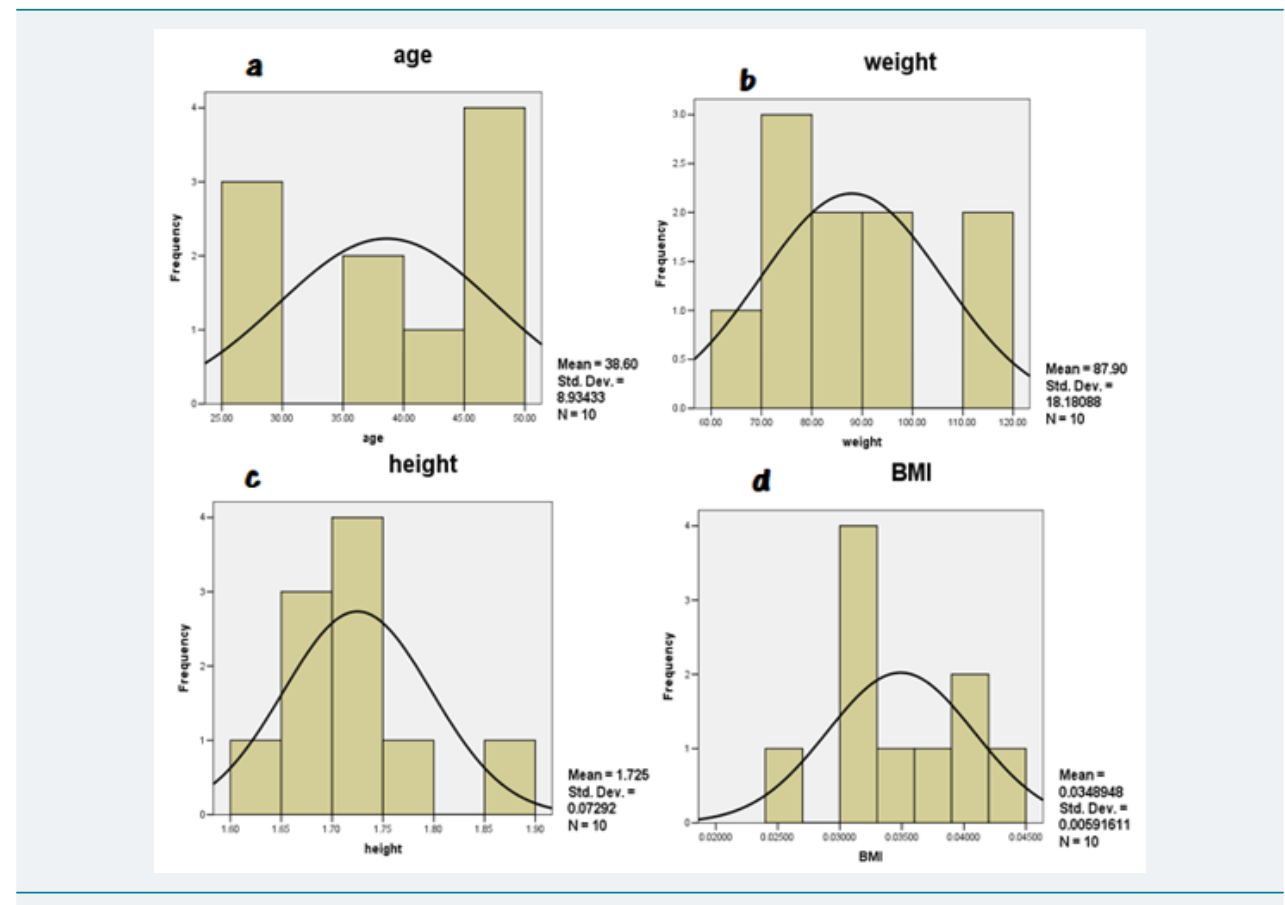

Figure 5: The sample in group B; (a) the main age; (b) main weight; (c) main height; and (d) the main BMI of the group.
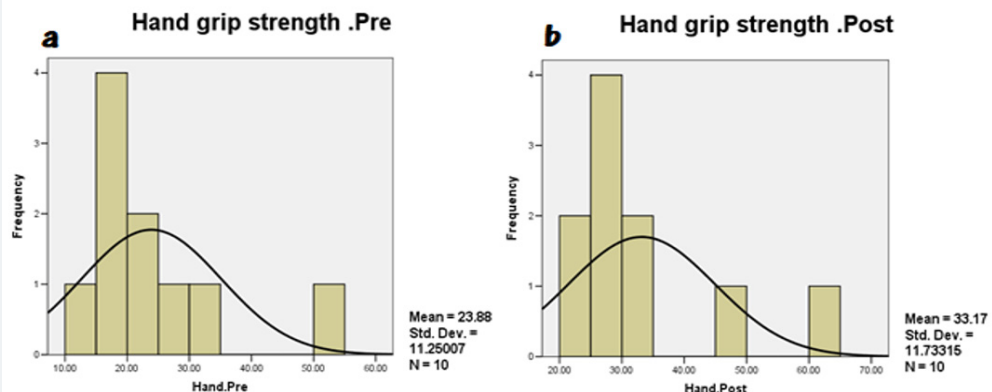

Figure 6: The effectiveness of the treatment for the hand grip in group $B$; (a) pre- treatment; and (b) post treatment.
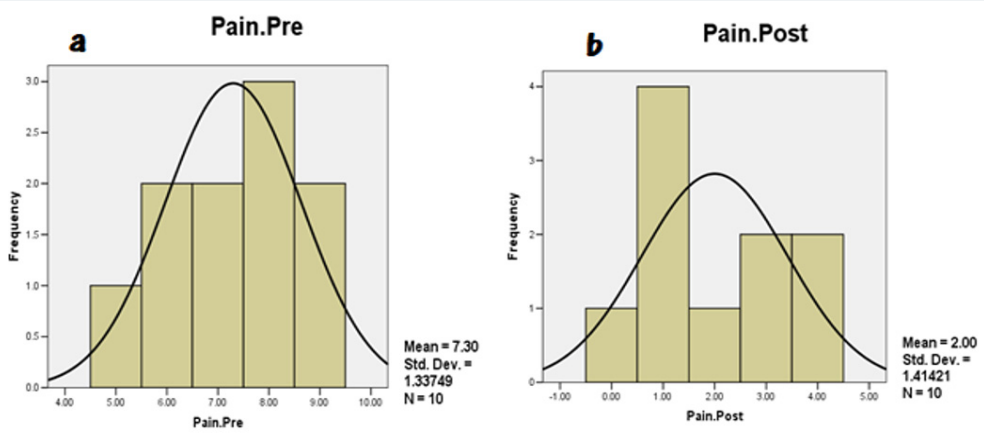

Figure 7: The differences of the pain for the group B between; (a) pre-treatment; and (b) post treatment. 


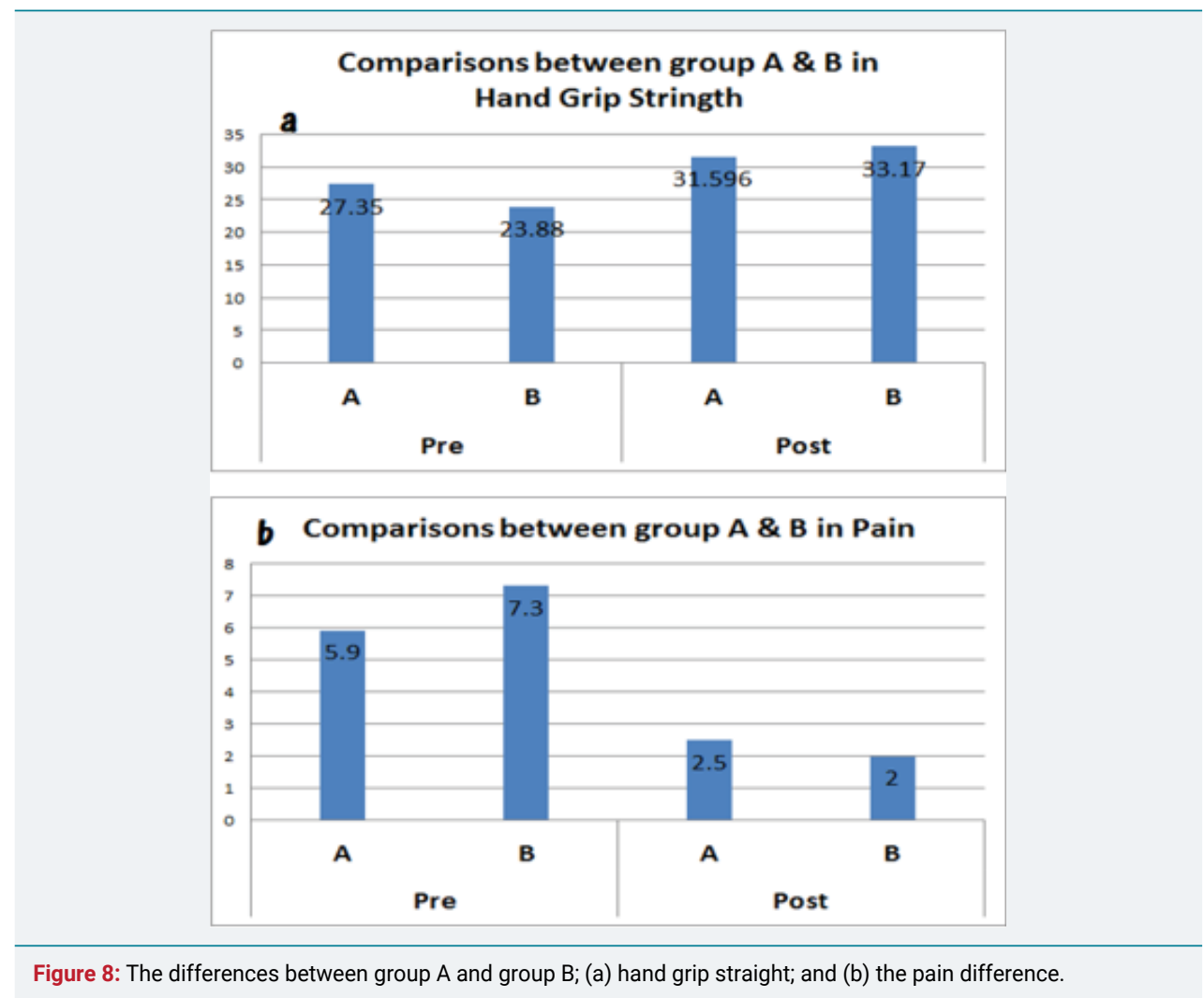

in patient with tennis elbow. Restoring full function in the wrist and the hand is the major goal of lateral epicondylitis rehabilitation.

The current study revealed that therapeutic ultrasound or kinesio taping combined with exercises for four weeks in patients with tennis elbow resulted in significant increase in hand grip strength in the post-treatment, associated with a significant decrease in pain intensity in the post-treatment in both groups. In addition, it revealed that there was significant difference between therapeutic ultrasound and kinesio taping as regard to pain reduction and grip strength improvement, as kinesio taping allows a greater degree of pain relief and strength improved than the therapeutic ultrasound. All patients in both groups had symptoms of tennis elbow, with pain over the lateral aspect of the elbow that is aggravated by wrist extension and direct palpation over the lateral epicondyle of the humorous.

From statistical analysis of pre and post treatment of pain assessment in patients who received ultrasound, there was a significant difference of pain intensity value between pre-treatment value and post-treatment value. Also, there was a significant difference of hand grip strength value between pre-treatment value and post-treatment value. To examine the effect the therapeutic ultrasound before and after treatment results of pain was measured with a visual analogue scale. There was significant decrease of pain intensity after treatment. The result of this study agreed with several studies which investigated the pain severity using visual analog scale [12].

This effect on pain can be explained as suggested by Wilkin [13] and Greg [14] ultrasound is vibration of the tissue causing microscopic bubbles to form, which transmit the vibrations in a way that directly stimulates cell membranes. This physical stimulation appears to enhance the cell-repair effects of the inflammatory response and thereby reducing pain.

The results of this study was supported with several studies reported that significant decrease in pain severity of tennis elbow patients using the ultrasound $[15,16]$. However, the study by D'Vaz [17], assessed the effectiveness of low-intensity 
ultrasound therapy (LIUS) vs placebo therapy daily for 12 weeks in patients with chronic lateral epicondylitis (LE) in a randomized, double-blind, placebo controlled trial. In this study LIUS was no more effective for a large treatment effect than placebo for recalcitrant LE. To examine the effect, the ultrasound on grip strength pre and post treatment using handheld dynamometer. There was significant improvement in grip strength after treatment.

Grip strength is considered the most valid outcome measure for lateral epicondylitis. At this study grip strength was assessed by handheld dynamometer. This finding was supported by the finding of $[18,19]$. The increased grip strength with using the ultrasound in tennis elbow patients may explained that ultrasound therapy may accelerate the healing process in damage tissues. These mechanisms may explain our finding that ultrasound decrease pain thus increase active movement of the wrist and grip strength [20].

From statistical analysis of pre and post treatment of pain assessment in patients received kinesio taping, there was a significant difference of pain intensity value between pre-treatment value and post-treatment value. Also, there was a significant difference of hand grip strength value between pre-treatment value and post-treatment value. To examine the effect kinesio taping pre and after treatment results of pain was measured with a visual analogue scale. There was significant decrease of pain intensity after treatment.

Alireza and Mohammad [4] reported that there were significant differences in wrist extension forces between effected and unaffected arms. Changes in grip strength showed statically significant improvements in the affected arm compared to the unaffected arm Also, in assessment of pain at the lateral epicondyle, the mean change between affected and unaffected arms was significant.

Comparing between the effect of therapeutic ultrasound and kinesio taping on lateral epicondylitis. After treatment there was significant difference between therapeutic ultrasound and kinesio taping as regard to pain reduction and grip strength improvement as kinesio taping allows a greater degree of pain relief and greater degree of grip strength improvement than the therapeutic ultrasound for patients with lateral epicondylitis.

In the current study, both groups treatment was combined with progressive stretching and strengthening exercises. Eccentric training results in tendon strengthening by stimulating mechano-receptors in tenocytes to produce collagen, which is probably the key cellular mechanism that determines recovery form tendon injuries. In addition, eccentric training may induce a response that normalizes the high concentrations of glycosaminoglycans. It may also improve collagen alignment of the tendon and stimulate collagen cross-linkage formation, both of which improve tensile strength. It has also been proposed that the positive effects of exercise programmes for tendon injuries may be attributable to either the effect of stretching, with a lengthening of the muscle-tendon unit and consequently less strain experienced during joint motion, or the effects of loading within the muscle-tendon unit, with hypertrophy and increased tensile strength in the tendon also during eccentric training, the blood flow is stopped in the area of damage and this leads to neovascularization, the formation of new blood vessels, which improves blood flow and healing in the long term.

\section{Conclusion}

The conclusion of the study is that the therapeutic ultrasound combined with exercises was effective in improving pain intensity and increasing hand grip strength. Kinesio tape combined with exercises was effective in improving pain intensity and increasing hand grip strength. There was significant difference between ultrasound 
and kinesio tape as regard to pain reduction and improvement grip strength where kinesio tape allows a greater degree of pain relief and greater improvement of grip strength than the ultrasound.

\section{References}

1. De Smedt $T$, de Jong A, Van Leemput W, Lieven $D$, Van Glabbeek F. Lateral epicondylitis in tennis: Update on aetiology, biomechanics and treatment. Br J Sports Med. 2007; 41: 11 816-819. Ref.: https://tinyurl.com/y3lrvw6p

2. Lee WH, Kwon OY, Yi CH, Jeon HS, Ha SM. Effects of Taping on Wrist Extensor Force and Joint Position Reproduction Sense of Subjects With and Without Lateral Epicondylitis. J Phys Ther Sci. 2011; 23: 629-634. Ref.: https://tinyurl.com/yyacpsw5

3. Cullinane FL, Boocock MG, Trevelyan FC. Is eccentric exercise an effective treatment for lateral epicondylitis? A systematic review. Clin Rehabil. 2014; 28: 3-19. Ref.: https://tinyurl.com/y2ewoel6

4. Shamsoddini A, Hollisaz MT. Effects of taping on pain, grip strength and wrist extension force in patients with tennis elbow. Trauma Mon. 2013; 18: 71-74. Ref.: https://tinyurl.com/y2uxpd56

5. van der Worp $\mathrm{H}$, van den Akker-Scheek I, van Schie H, Zwerver J. ESWT for tendinopathy: Technology and clinical implications. Knee Surgery Sport Traumatol Arthrosc. 2013; 21: 1451-1458. Ref.: https://tinyurl.com/y454965g

6. Galloway MT, Lalley AL, Shearn JT. The Role of Mechanical Loading in Tendon Development, Maintenance, Injury, and Repair. J Bone Joint Surg Am. 2013; 1620-1628. Ref.: https://tinyurl.com/y4jo59kx

7. Robertson C, Saratsiotis J. A review of compressive ulnar neuropathy at the elbow. J Manipulative Physiol Ther. 2005; 28: 345. Ref.: https://tinyurl.com/y332az2b

8. Jafarian FS, Demneh ES, Tyson SF. The Immediate Effect of Orthotic Management on Grip Strength of Patients With Lateral Epicondylosis. J Orthop Sport Phys Ther. 2009; 39: 484-489. Ref.: https://tinyurl.com/y3tvr4jx

9. Kachanathu SJ, Miglani S, Grover D, Zakaria AR. Forearm Band Versus Elbow Taping: As a Management of Lateral Epicondylitis. J Musculoskelet Res. 2013; 16: 1350003. Ref.: https://tinyurl.com/y6lc29n8

10. Zhu J, Hu B, Xing C, Li J. Ultrasound-guided, minimally invasive, percutaneous needle puncture treatment for tennis elbow. Adv Ther. 2008; 25: 1031-1036. Ref.: https://tinyurl.com/yyqhetqw

11. Stasinopoulos D, Stasinopoulos I, Pantelis M, Stasinopoulou K. Comparison of effects of a home exercise programme and a supervised exercise programme for the management of lateral elbow tendinopathy. Br J Sports Med. 2010; 44: 579-583. Ref.: https://tinyurl.com/y47e373b

12. Green $S$, Buchbinder R, Barnsley L, Hall S, White M, et al. Acupuncture for lateral elbow pain. Cochrane Database Syst Rev. 2002; CD003527. Ref.: https://tinyurl.com/y5pr9tf3

13. Watson T. Ultrasound in contemporary physiotherapy practice. Ultrasonics. 2008; 48: 321-329. Ref.: https://tinyurl.com/yx9l|cjv

14. Varghese S. A comparative study of Mulligan's mobilisation with movement with and without low level under the guidance of without level laser. MVS College. 2010. Ref.: https://tinyurl.com/yytbd98a

15. Bisset L, Paungmali A, Vicenzino B, Beller E. A systematic review and meta-analysis of clinical trials on physical interventions for lateral epicondylalgia. Br J Sports Med. 2005; 39: 411-422. Ref.: https://tinyurl.com/y5z2wnlc

16. Bisset L, Beller E, Jull G, Brooks $P$, Darnell R, et al. Mobilisation with movement and exercise, corticosteroid injection, or wait and see for tennis elbow: Randomised trial. Br Med J. 2006; 333 939-941. Ref.: https://tinyurl.com/y5fcfe2z

17. D'Vaz A, Ostor AJ, Speed CA, Jenner JR, Bradley M, et al. Pulsed low-intensity ultrasound therapy for chronic lateral epicondylitis: A randomized controlled trial. Rheumatology. 2006; 45: 566-570. Ref.: https://tinyurl.com/yxfmc6h9

18. Stasinopoulos D, Johnson MI. Cyriax physiotherapy for tennis elbow/lateral epicondylitis. Br J Sports Med. 2004; 38: 675-677. Ref.: https://tinyurl.com/y5omnrpj

19. Kochar M, Dogra A. Effectiveness of a specific physiotherapy regimen on patients with tennis elbow: Clinical study. Physiotherapy. 2002; 88: 333-341. Ref.: https://tinyurl.com/y3kpaor4

20. Bakhtiary $\mathrm{AH}$, Rashidy-Pour A. Ultrasound and laser therapy in the treatment of carpal tunnel syndrome. Aust J Physiother. 2004; 50: S147-151. Ref.: https://tinyurl.com/y4k72h4r 